\title{
The effect of perceptual organization on risky choice and numerical decisions
}

shows inter-subject correlation

Moshe Glickman ${ }^{1}$, Tal Sela ${ }^{2}$, Marius Usher ${ }^{1,4}$, Dino J Levy ${ }^{3,4 *}$

1) The School of Psychological Sciences, Tel Aviv University, Israel

2) School of Social Sciences and Humanities, Kinneret Academic College, Israel

3) Coller School of Management, Tel Aviv University

4) Sagol School of Neuroscience, Tel Aviv University

Contact information:

Moshe Glickman

glickman@mail.tau.ac.il

Tal Sela

ta19.sela@gmail.com

Marius Usher

marius@tauex.tau.ac.il

Dino J Levy

dinolevy@tauex.tau.ac.il

* Corresponding author 


\title{
The effect of perceptual organization on risky choice and numerical decisions shows inter-subject correlation
}

\author{
Moshe Glickman ${ }^{1}$, Tal Sela ${ }^{2}$, Marius Usher ${ }^{1,4}$, Dino J Levy ${ }^{3,4 *}$
}

\begin{abstract}
Individual differences in cognitive processing have been the subject of intensive research. An important type of such individual differences is the tendency for global vs. local processing, which was shown to correlate with a wide range of processing differences in fields such as decision making, social judgments and creativity. Yet, whether these global/local processing tendencies are correlated within a subject across different domains is still an open question. To address this question, we develop and test a novel method to quantify global/local processing tendencies, in which we directly set in opposition the local and global information instead of instructing subjects to specifically attend to one processing level. We apply our novel method to two different domains: i) a numerical cognition task, and ii) a preference task. Using computational models, we accounted for classical effects in choice and numerical-cognition. Global/local tendencies in both tasks were quantified using a salience parameter. Critically, the salience parameters extracted from the numerical cognition and preference tasks were highly correlated, providing support for robust perceptual organization tendencies within an individual.
\end{abstract}

\section{Introduction}

A fundamental question in perception is whether humans attend to a visual scene as a whole or whether they focus on its details. A visual object can be viewed as a multilevel hierarchical structure of parts and wholes (Palmer, 1977). Global/local processing reflects the priority of visual processing between these two levels (Navon, 1977; Wagemans, Elder, et al., 2012; Wagemans, Feldman, et al., 2012). While earlier studies have looked at group effects (e.g., Navon global-precedence theory (Navon, 1977)), more recent studies have focused on individual differences in global/local processing tendencies (Chamberlain, Van der Hallen, Huygelier, Van de Cruys, \& Wagemans, 2017), which are thought to relate with narrow vs. broad attentional focus (Dale \& Arnell, 2010, 2013, 2015). Moreover, it has been previously shown that global/local processing style varies as a function of experience (Caparos, Linnell, Bremner, de Fockert, \& Davidoff, 2013), culture (Caparos et al., 2012; Davidoff, Fonteneau, \& Fagot, 2008), and skilled behavior (Lewis \& Dawkins, 2015), suggesting that there is a large variation across individuals in the tendency for global/local processing. Recently, individual differences in global vs. local processing have been suggested to underlie a wide range of differences in cognition, in fields as diverse as decision-making, social-judgments, and creativity (Chamberlain, McManus, Riley, Rankin, \& Brunswick, 2013; Dijkstra, van der Pligt, \& van Kleef, 2013; Drake \& Winner, 2011; Förster, Liberman, \& Kuschel, 2008; Stoesz, Jakobson, Kilgour, \& Lewycky, 2007; Zmigrod, Zmigrod, \& Hommel, 2015). Furthermore, such differences are suggested to account for some of the cognitive pathologies in autism (De Martino, Harrison, Knafo, Bird, \& Dolan, 2008; Van Eylen, Boets, Steyaert, Wagemans, \& Noens, 2018) and schizophrenia (Bellgrove, Vance, \& Bradshaw, 2003).

Many studies have used the Navon task (Navon, 1977, 1981) to quantify individual differences in global/local processing style (Dijkstra et al., 2013; Förster \& Higgins, 2005; Hedge, Powell, \& Sumner, 2018). In this task, subjects are presented with hierarchical letters (e.g., small Ss in the shape of a large $\mathrm{H}$, and the other way around) and are asked to categorize based on either the global or the local shape (and ignore the other shape, which is designated as task irrelevant). In a recent study, Hedge et al., (2018) have examined test-retest reliability in several global-local indices extracted from the Navon task, such as the difference in reaction time between conditions in which attention was deployed to the local level (RT local) and to the global level (RT global). They reported low test-retest reliability as there was no 
significant correlation in this global/local index across the two sessions. Interestingly, there was another index that did show high test-retest reliability: the fraction of errors on only the local task. However, such a measure does not contrast local processing with global processing, and is very likely confounded with the ability of the subject to focus attention on the task at hand (local) and filter out the irrelevant dimension (global) (Mevorach, Humphreys, \& Shalev, 2006). Moreover, Milne and Szczerbinski (2009) showed that there were relatively low inter-subject correlations across 14 different tasks that were aimed to measure global/local processing style. Dale and Arnell (2013) came to a similar conclusion in which test-retest reliability across three different global/local processing tasks was relatively low. Finally, it was shown that global/local processing style as measured using two variations of the Embedded Figures Test (EFT) (Mumma, 1993; Witkin, H, Oltman, P, Raskin E \& Karp, 1971) showed low test-retest reliability with global/local processing style as measured using the Navon task (Chamberlain et al., 2017).

Hence, from decades of studies of global/local processing style, it seems that each specific task is measuring something slightly different compared to other tasks. Therefore, if one wants to examine if global/local processing style is robust across two different cognitive processes (for example numerical processing and value-based choice) it is important to use the same stimuli in both domains. Otherwise, a lack of correlation might be due to differences in task design, the exact construct that the task is measuring, and most importantly, in the way the perceptual manipulation is affecting behavior in each task.

Finding such a correlation will strengthen the notion that perceptual processes can affect different cognitive processes in a similar way within an individual. To this end, we focus here on the mechanism of spontaneous attentional selection to global/local information in the same hierarchical structure, and examine its impact on both tasks. Therefore, in the current study, we aim to examine to what extent subjects are influenced by global/local perceptual information in both a numerical and a preference task. In addition, we want to examine if there is an inter-subject correlation across these two tasks. Importantly, we use the exact same stimuli in both tasks, because different types of stimuli, may induce differences in the global/local processing that could mask what could otherwise be robust individual differences. We propose a novel method (based on Navon stimuli) to quantify individual differences in global/local tendencies in a numerical and a preference task. The key difference in our novel method is that, we do not set one of the stimuli (global or local level) as relevant and the other as irrelevant, which is the case in the standard procedure (Navon, 1977, 1981). Rather, we make both stimulus levels taskrelevant, by asking subjects to carry out a type of comparison between them. Thus, subjects had to perceive and attend to both levels of the hierarchical stimuli at the same time. This enabled us to directly contrast and examine each subject's tendency to spontaneously focus on either the global or the local features of each stimulus, under the rationale that if a subject has a tendency towards global or local processing, this should be reflected in the relative salience of the global/local information when both are task relevant. To extract for each subject this global-local salience tendency parameter, we carried out computational modelling.

We implemented our novel procedure in two domains: i) a numerical cognition task (numerosity comparison), and ii) a mixed-gambles preference task (with gain/loss gambles). Each subject performed these tasks in two separate sessions one week apart. We then utilized the individual differences in these tasks and correlated, across subjects, between the global/local tendencies estimated from each of the tasks. 


\section{Materials and Methods \\ Subjects}

Thirty subjects were recruited for the current study (mean age=23.25, SD=4.09; 16 female), in exchange for course credits. Their sight was normal or corrected to normal. All subjects gave written informed consent. All methods were performed in accordance with the relevant guidelines and regulations of the declaration of Helsinki. All experiments were approved by the committee for the protection of human subjects at Tel Aviv University.

\section{Stimuli and apparatus.}

The stimuli were exactly the same in both tasks. Subjects sat in a dark room and the stimuli were presented on a 17 -inch monitor (1280X1024 pixels) with a refresh rate of $75 \mathrm{~Hz}$. The viewing distance was $70 \mathrm{~cm}$, so that each centimeter on the screen represented $0.81^{\circ}$ of visual angle. All stimuli appeared against a black background. Each compound figure was spatially arranged on a $5 \times 7$ grid to form a global number that subtended $3.16^{\circ} \times 6^{\circ}$ of visual angle (in width and height, respectively). Each local number subtended $0.35^{\circ} \times 0.56^{\circ}$ of visual angle (in width and height, respectively). The inter-element distance was $0.35^{\circ}$. The compound stimuli could appear at one of two possible locations with a distance of $1.50^{\circ}$ to the left or right of the fixation mark along the midline. The mask was a $5 \times 7$ grid of filled white dots, subtended identical size for both local and global elements as the target stimulus. The experiment was run using E-Prime v.2.

\section{Session 1: Numerical cognition task}

\section{Materials and procedure.}

We developed a behavioral paradigm based on Navon's figure (Navon, 1977) that included numbers instead of letters (cf. Della Libera \& Chelazzi, 2006). The stimuli were composed of a combination of all possible pairs of numbers between 2 and 9 (excluding combinations in which the global and the local values were equal because neither of the levels is larger) for a total of 56 unique combinations. For each compound figure, we computed the numerical distance by calculating the absolute difference between the global and the local numbers (numerical distance). In addition, we defined a congruent (incongruent) trial, as those in which the number at the global (local) level was larger than the number at the local (global) level (Fig. 1A). On each trial, subjects were instructed to look at the compound figure and decide whether the number appearing at the global level or at the local level was larger. Fig. 1C illustrates the timeline of the experiment. We recorded RTs and accuracy for each response. The task included 448 trials (split to four blocks of 112 trials each). Within each block, the order of stimuli presentation was random. Each unique compound figure was presented eight times in total. After subjects completed the session, we gave them an endowment of $\mathrm{v} 40$ (equivalent to $\sim \$ 12$ ) and asked them to bring this amount of money to the second session.

\section{Session 2: Mixed-gambles task}

\section{Materials.}

We used the exact same compound figure as in session 1. However, as can be seen in Fig. 1B, each compound figure represented a 50-50 chance of winning or losing some amount of money against a fixed value of zero. We used the same number matrix as in the first task but we also included the set of all equal-number trials (e.g., large 5 composed out of small 5s) for a total of 64 unique compound figures. As shown in Fig.1D, the risky gamble in each trial had 1 of 8 potential gains ranging from $+\$ 2$ to $+\$ 9$, and 1 of 8 potential losses ranging from $-\$ 2$ to $-\$ 9$, both sampled in increments of $\$ 1$. Although the different lotteries were expressed in US dollars, it was clear to our subjects that they are playing for 
Israeli shekels. That is, we multiplied by 4 all payoffs (dollar-shekel exchange rate) at the time of payment.

For each lottery (compound figure), we computed the expected value (EV) as follows:

$$
E V_{i}=p \times \text { Amount }_{G(i)}+(1-p) \times \text { Amount }_{L(i)}
$$

where $E V_{i}$ is the expected value for trial $i$, Amount $_{G(i)}$ and Amount $_{L(i)}$ are the monetary amounts that can be gained or lost, respectively, and $p$ is the probability of winning (always 0.5 in our experiment).

A

B

\begin{tabular}{|c|c|c|c|c|c|c|c|}
\hline \multicolumn{3}{|c|}{ Perceptual-numerical task } & \multicolumn{5}{|c|}{ Mixed-gambles task } \\
\hline$\underline{\text { Stimuli }}$ & Congruency & $\frac{\text { Numerical }}{\text { Distance }}$ & \# & Condition & Lottery & $\begin{array}{c}\text { Number } \\
\text { (Global, Local) }\end{array}$ & $\underline{E V}$ \\
\hline $\begin{array}{lr}22222 \\
2 & 2 \\
2 & 2 \\
2222 & 2 \\
& 2 \\
2222 & 2 \\
222 & \end{array}$ & Congruent & 7 & (1) & GLLG & $\begin{array}{l}\mathbf{5 0} \% \text { to gain } \mathbf{\$ 9} \\
\mathbf{5 0} \% \text { to loss } \mathbf{\$ 2} \\
\mathbf{5 0} \% \text { to gain } \$ 2 \\
\mathbf{5 0} \% \text { to loss } \mathbf{\$ 9}\end{array}$ & $(9,2)$ & +3.5 \\
\hline $\begin{array}{l}99999 \\
9 \\
9 \\
99999 \\
9 \\
9 \\
99999\end{array}$ & Incongruent & 7 & (3) & GLLG & $\begin{array}{l}\mathbf{5 0} \% \text { to gain } \$ 2 \\
50 \% \text { to loss } \$ 9 \\
50 \% \text { to gain } \$ 9 \\
50 \% \text { to loss } \$ 2\end{array}$ & $(2,9)$ & $\begin{array}{r}-3.5 \\
+3.5\end{array}$ \\
\hline
\end{tabular}

C

D
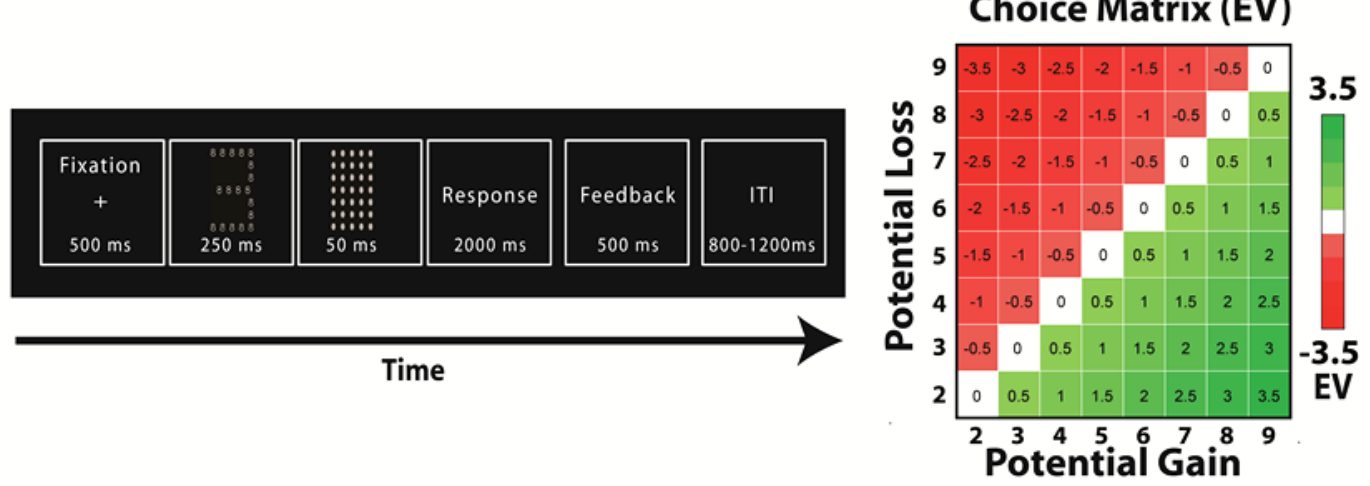

Figure 1. Experimental design. (A) Stimuli: An illustration of the compound figure used for both tasks. Features of the Numerical cognition task. Each stimulus was characterized by Congruency: a description of the example stimulus if it was Congruent (the global level is numerically bigger than the local level) or Incongruent, and by Numerical Distance: the numerical difference between the global and local levels ('7' in the example stimulus). (B) Features of the Mixed-Gambles task. Condition: The task included two conditions: (1) the number in the global level represented the potential gain amount, whereas the number in the local level represented the potential loss amount (GGLL); and (2) vice versa, in which the number in the global level represented the potential loss amount, whereas the number in the local level represented the potential gain amount (GLLG). Lottery: a description of the possible gambles based on the condition. EV: the expected value of the example lottery in each of the conditions. $(\boldsymbol{C})$ Single-trial procedure for both tasks. (D) The choice matrix representing all the lotteries and their corresponding EVs that were used in the mixed-gambles task. 


\section{Procedure.}

A week after the perceptual-numerical task, subjects came to the lab again and conducted the mixedgambles task. Subjects were asked to accept or reject a series of mixed gambles with equal probability $(50 \%)$ of winning or losing a variable amount of money against a fixed amount of zero. Subjects responded by a key press on the keyboard. The timeline was identical to the timeline of the perceptualnumerical task (Fig. 1C). However, in the beginning of each block we informed the subject regarding the condition type of that block (Global Gain-Local Loss; GGLL), or vice versa (Global Loss-Local Gain; GLLG). That is, whether the global (local) level represented a gain or a loss. Subjects completed a total of 512 trials, 256 trials per condition. There were four blocks (128-trial per block) with two blocks for each condition. The order of block presentation was counterbalanced across subjects. Within each block, the order of lottery presentation was random. Overall, each unique lottery was presented eight times, four times for each condition. To introduce incentive-compatible payoffs, our subjects were endowed with $\mathrm{v} 40$ (equivalent to $\sim$ 12) on the first session (at the end of the numerical cognition task), and knew that at the end of the experiment, the computer would randomly pick one trial and they could win, lose, or stay with the endowment they received based on the choice they have made on that trial.

\section{Computational Modelling}

1. Preference-task - The probability to accept/reject the lottery was calculated using the following equations:

$$
\begin{gathered}
p(\text { accept })=\frac{1}{1+e^{-(\beta \cdot U(\text { lottery }))}} \\
p(\text { reject })=1-p(\text { accept })
\end{gathered}
$$

where $\beta$ is a free parameter indicating sensitivity to utility (i.e., inverse of noise), and the utility of each lottery was defined as:

$$
U(\text { lottery })= \begin{cases}\text { Amount }_{G} \cdot \theta_{\text {preference }- \text { Amount }_{L} \cdot \lambda,} & \text { GGLLcondition } \\ \text { Amount }_{G}-\text { Amount }_{L} \cdot \lambda \cdot \theta_{\text {preference }}, & \text { GLLGcondition }\end{cases}
$$

where Amount $_{G}$ and Amount $_{L}$ represent the gain and loss amounts, respectively, $\lambda$ is a loss aversion parameter (Tversky \& Kahneman, 1992), and $\theta_{\text {preference }}$ is a free parameter representing the value modulation resulting from global/local biases, such that $\theta_{\text {preference }}>1$ indicates a global-bias, $\theta_{\text {preference }}<1$, indicates a local-bias, and $\theta_{\text {preference }}=1$, indicates a lack of any global/local bias. Thus, if a subject has a global bias, values presented as global numbers (either gains or losses) are increased by a factor $\theta$, and values presented as local numbers are reduced by the same factor. If a subject has no global/local bias, the factor is equal to 1 , (and thus the value function is similar to the standard Prospect Theory function with $\alpha=1$ (Kahneman $\&$ Tversky, 1979).

2. Numerical Cognition task - The performance in the numerical cognition task was modeled using a variant of the neural network model developed by Verguts, Fias, \& Stevens (2005) The model has three layers (Fig. 2):

(i) Input layer, which is consisted of a set of units corresponding to Arabic numbers based on place-coding system (the number "1" activates the first unit, the number "2" activates the second unit, and so on).

(ii) Hidden layer, which is also consisted of units corresponding to Arabic numbers based on a place-coding system. This layer is fed with the unit activated in the first layer, and activates the corresponding unit with maximal strength, as well as neighboring units which are activated with gradually decreasing strength as a function of distance. 
(iii) Response layer, which consists of two units corresponding to the global/local responses. The response layer is fed with the activations of the units in the second layer, and accumulates their values across time as in the Drift Diffusion Model (Ratcliff, 2002; Ratcliff \& McKoon, 2008; Ratcliff \& Rouder, 1998). A response is initiated once the difference between the global and local units crosses a predefined threshold.

In order to model global/local biases, we multiplied the input to the first layer by a free parameter $\theta_{\text {perceptual }}$. Similar to the preference-task model: $\theta_{\text {perceptual }}>1$ indicates a global-bias, $\theta_{\text {perceptual }}<1$ indicates a local-bias, and $\theta_{\text {perceptual }}=1$, indicates lack of bias.

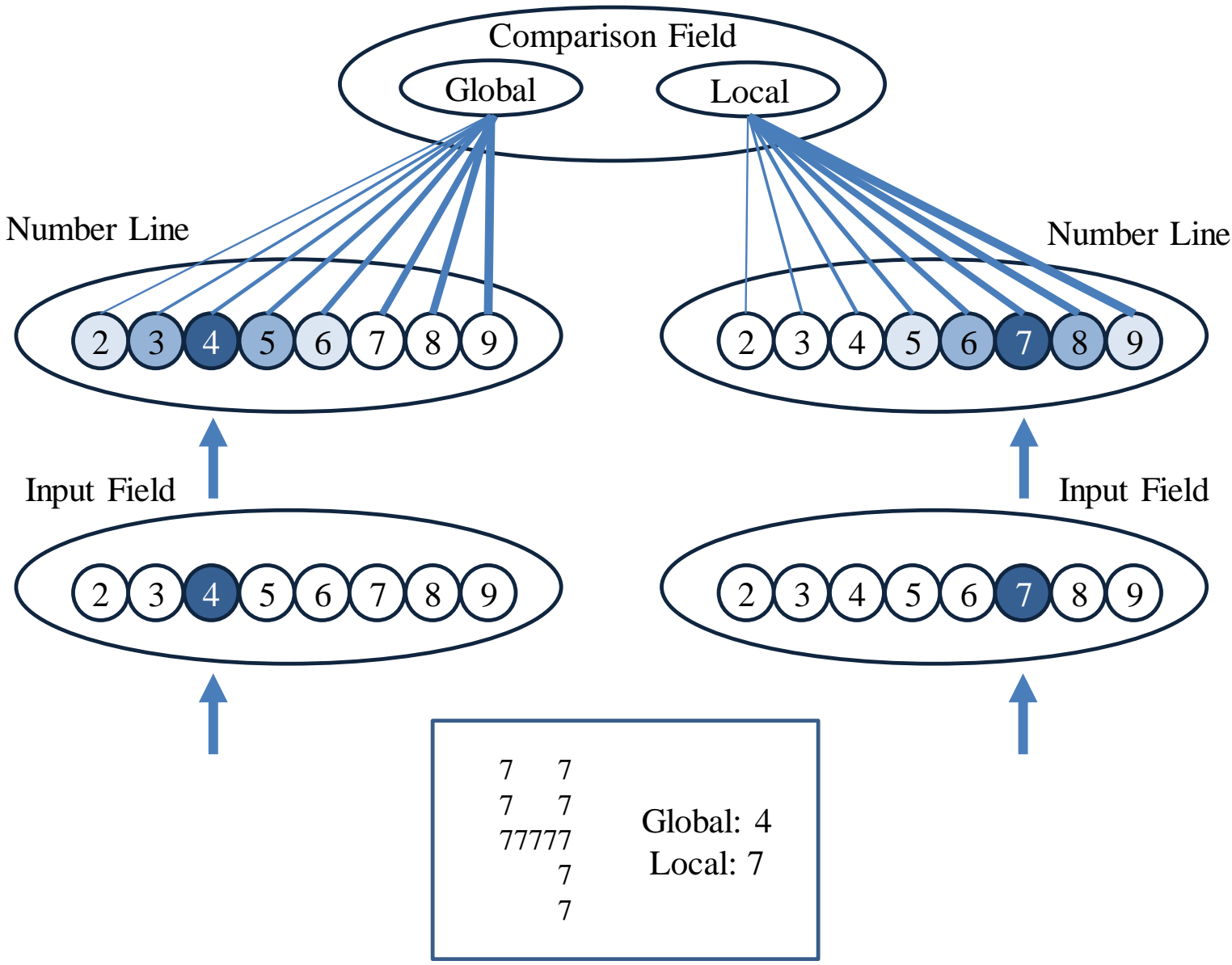

Figure 2. Computational model for the Numerical Cognition Task. The first (bottom) layer is the input layer, which corresponds to Arabic numbers based on a place-coding system. The second (middle) layer is a hidden layer, which activates the corresponding unit with maximal strength, and neighboring units with gradually decreasing strength as a function of distance. The third (top) layer is the response layer according to the global/local information. Blue lines correspond to the connections between the second to third layer; Lines thickness is proportional to connection strength. Adapted based on Verguts el al. (2005). 


\section{Results}

\section{Numerical Cognition task}

In the numerical cognition task, we found the standard distance effects: RTs were faster $(F(2,58)=$ $101.72, p<.001)$ and accuracy $(F(2,58)=11.94, p<.001)$ was higher when the two digits (at the global and local levels) were more distant from each other (e.g., 2-9 compared with 4-5; Fig. 3A). Furthermore, most subjects showed a congruency effect (global precedence effect): more correct responses and faster RTs when the global digit is larger than the local digit, compared to the opposite. This effect, however, was subject to considerable individual differences as shown in Figure 3B. Next, we fitted the computational model of numerical cognition (Verguts et al., 2005) to the data (see Table S1 for the best fitted parameters of each participant). As can be seen, the model captured well the RT-distributions (see Fig. 5C-D for two representative participants and Fig. S1 for all participants), and qualitatively accounted for the distance (Fig. 3E) and congruency effects (Fig. 3F) for accuracy and response-times.

\section{Mixed-gamble task}

In the mixed-gamble task, participants showed sensitivity to the EV of the mixed gambles. A mixedeffect logistic regression on the choice data, with the EV of the mixed-gamble as a predictor and with random intercepts and slopes at the participant level, showed that the preference for the mixed gamble over the zero-default option increased with the EV of the lottery $(\beta=1.06, p<.001$; Fig. 4A). In addition, participants show a typical loss-aversion behavior, which was measured using two methods: i) the EV value at the indifference point (method 1 , Fig 4B/top), $t=2.42, p=.021$, and ii) the overall acceptance rate for the gamble option (method 2, Fig. 4B/bottom), $t=2.34, p=.026$. Both measures show a very high correlation between them $(r=0.97, p<.001)$, but were subject to high individual differences (Fig. 4 C).

Our central focus is to examine individual differences in the level of their global/local bias (i.e., their tendency to demonstrate a global precedence effect). We extracted these biases in two ways: i) a simple data driven method, and ii) a computational method. The data driven method, extracts for each subject the fraction of trials, in which s/he responded on the basis of the global level, i.e., the fraction of trials in which the subject accepted the gamble when the global number represented a gain, and rejected it when the global number represented a loss (independently of the actual magnitude of the gain or loss). The computational method, fits to each subject a salience to global (bias) parameter, $\theta$ which is larger than 1 for an individual with a global-bias and smaller than 1 for an individual with a local bias (see Computational models). While on average at the group level, there was only a small global/local bias $\left(\theta_{\text {median }}=1.001\right)$, we found large individual differences (Fig. 4D; see Table S2 for the best fitted parameters of each participant), which were highly correlated between the data-driven and computational methods (Fig. 4E). Importantly, no correlation was observed between the individual tendency for loss-aversion and for global/local bias $(r=0.18, p=0.35$; Fig. 4F), which suggests that these biases stem from different underlying cognitive mechanisms. 
(A)
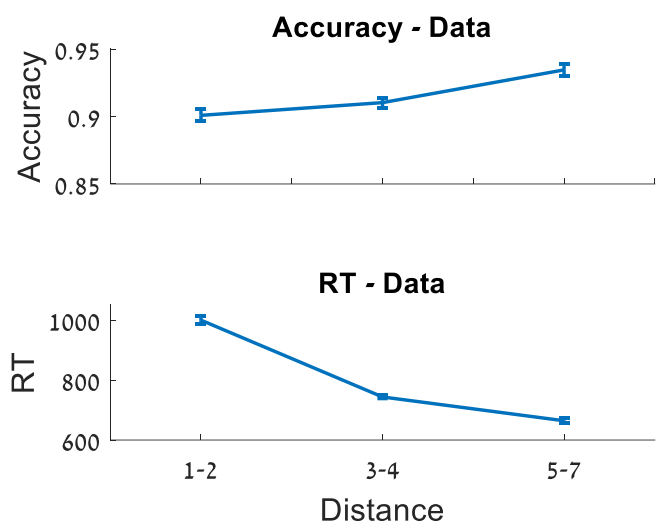

(C)

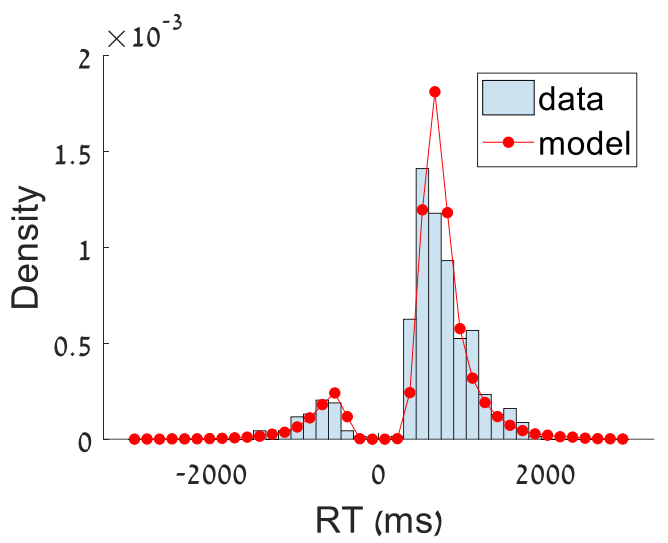

(E)
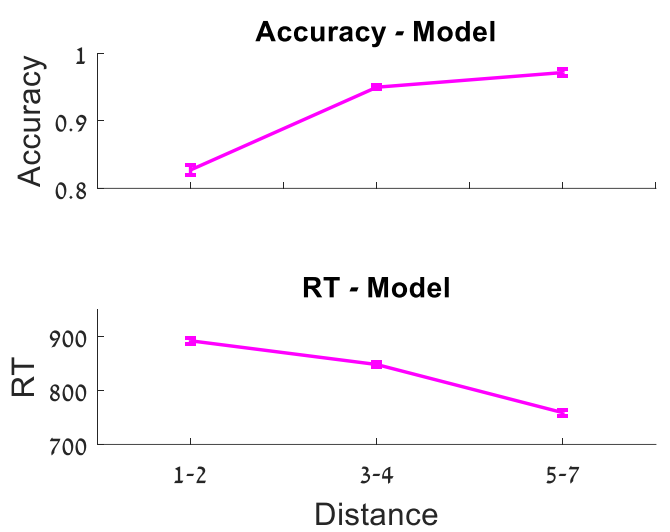

(B)
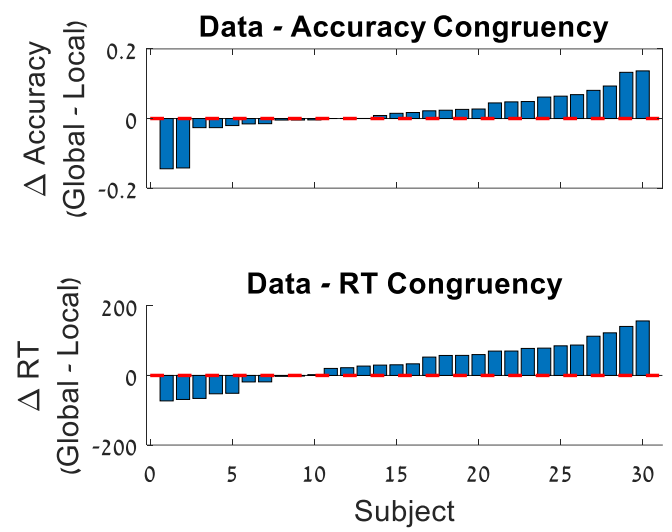

(D)

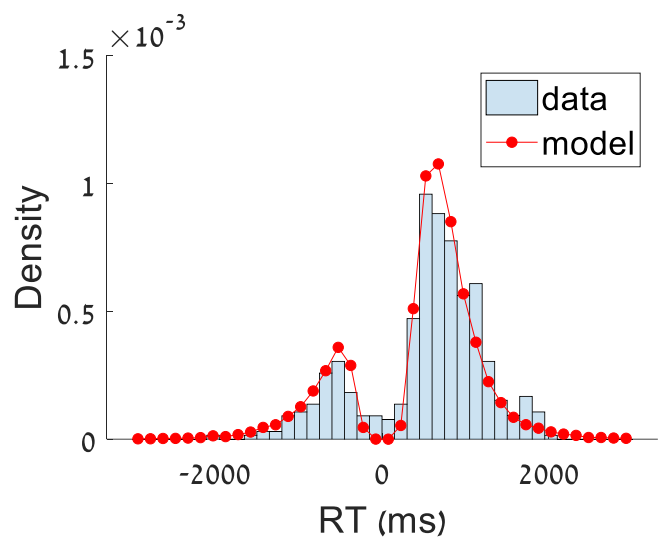

(F)
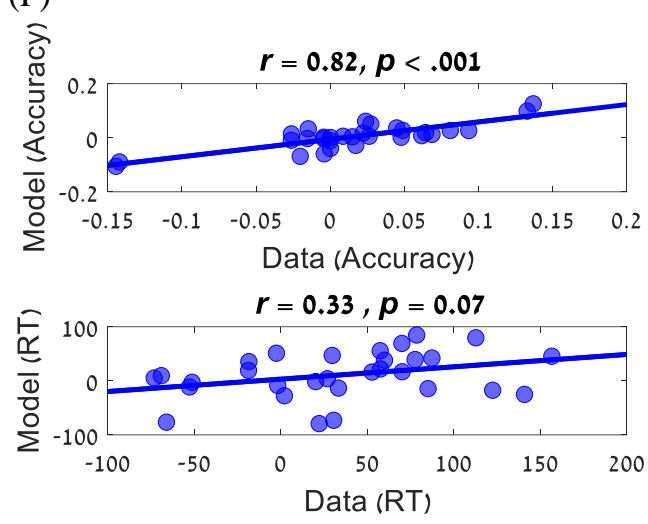

Figure 3. Numerical cognition task. (A) Distance effects in the numerical cognition task for accuracy and RT. Error bars correspond to within-subject standard errors of the mean. (B) Individual differences in congruency effects in the numerical cognition task for accuracy and RT. Subjects in the two panels are sorted independently. (C-D) Response-Time distributions of two representative participants. The $x$-axis indicates Reaction-Times, and the y-axis indicates the density. Error responses are mirrored on the negative $x$-axis. Red lines represent simulated RT-distributions for each participant, based on 1,000 simulations of each trial. (E) Model predicted distance effects in the numerical cognition task for accuracy and RT. Error bars correspond to within-subject standard errors of the mean. (F) Correlations between the empirical (a-axis) and predicated (y-axis) congruency effects for accuracy (top panel) and RT (bottom panel). Each point corresponds to a single participant. 
(A)

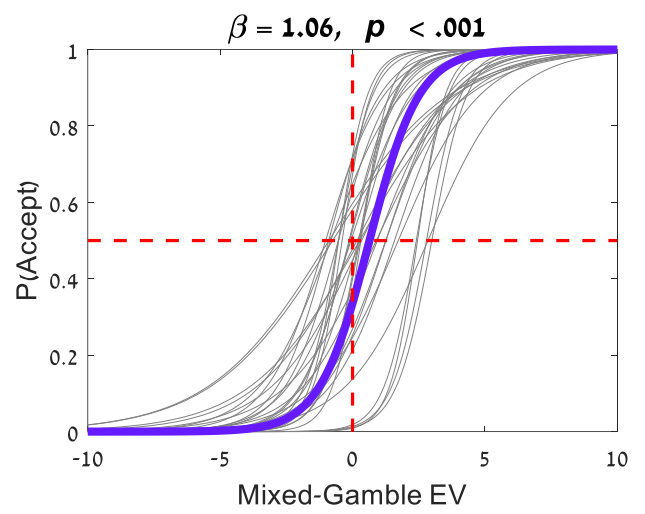

(C)

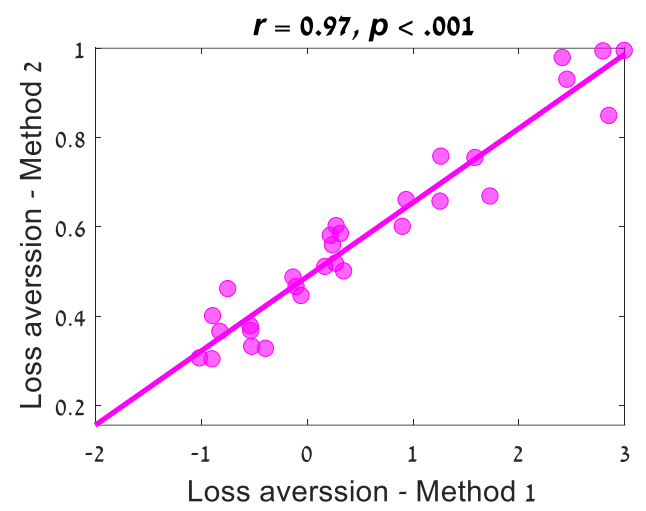

(E)

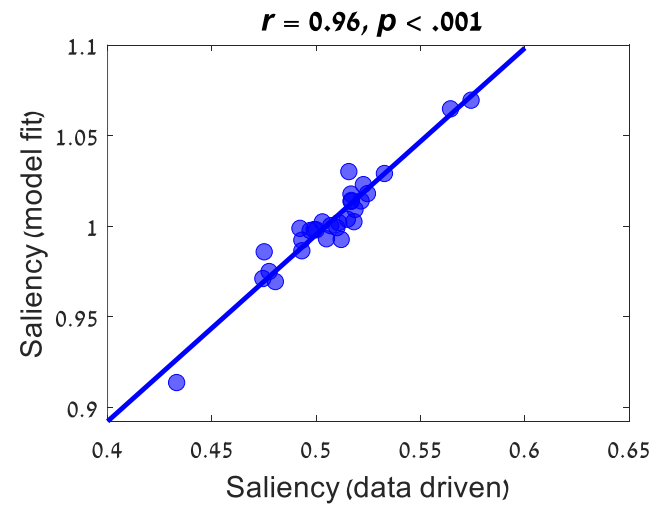

(B)
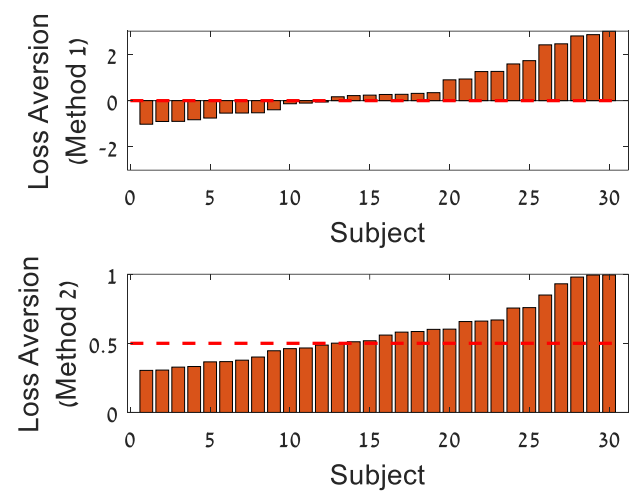

(D)
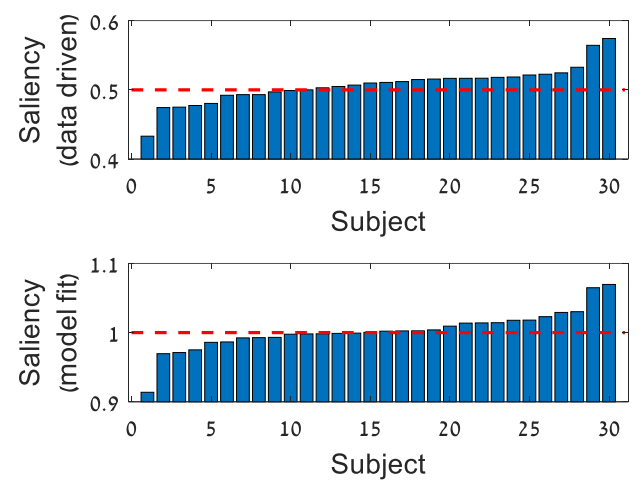

(F)

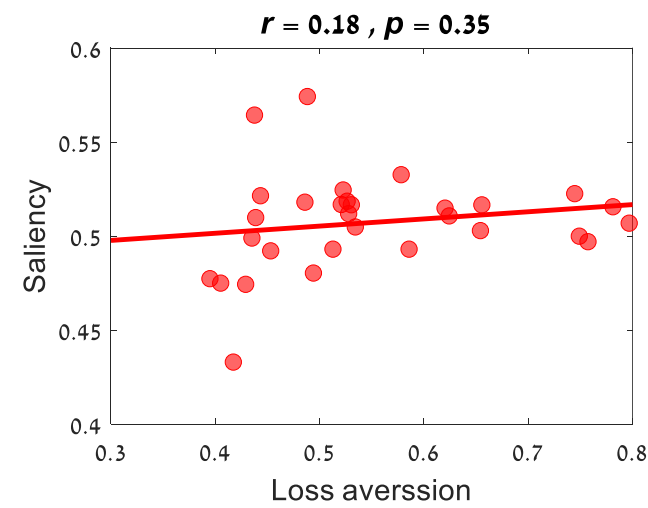

Figure 4. Preference task. (A) Participants were sensitive to changes in EV and demonstrate lossaversion at the group-level (the EV value at indifference point is higher than 0); Solid purple line corresponds to the group fit; grey lines correspond to the fit of individual participants. (B) Individual differences in loss-aversion, estimated as rejection rate for $E V=0$ (Method 1; upper panel), and as the acceptance rate for the gamble (Method 2; lower panel); Red dashed lines indicate loss-neutrality. Subjects in the two panels are sorted independently. (C) Correlation between loss-aversion as estimated using both methods. (D) Individual differences in data driven global-local bias (upper panel), and global-local bias based on a computational method model fit (lower panel); Red dashed lines indicate lack of bias. (E) Correlation between global-local bias as estimated using both methods (data driven and model fit). (F) No correlation was found between the global-local bias and loss aversion. 


\section{Across domain global-local individual differences}

The central aim of our study was to test for correlations between measures of global/local processing across the perceptual and preference domains. As shown in Fig. 5, there is a robust correlation between the individual differences in the two domains. This is shown both via the computational based estimated saliency parameters (Fig. 5A), as well as using the data driven approach, in which we correlated the salience effect in preference with the salience effect in perceptual choice (quantified as accuracycongruency in the numerical cognition task; Fig. 5B).

(A)

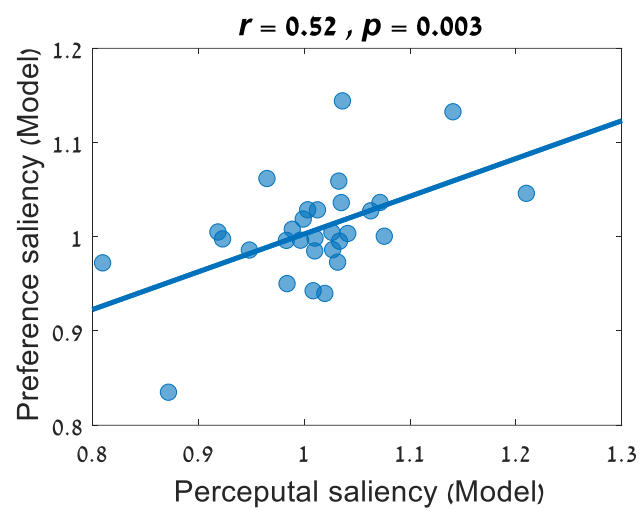

(B)

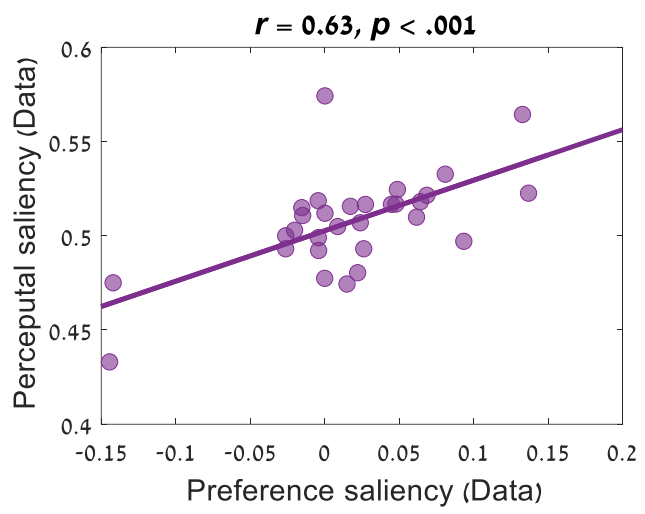

Figure 5. Correlations of global/local processing style between the perceptual and preferential tasks. (A) Model driven approach: The perceptual saliency parameter $\left(\theta_{\text {perceptual }}\right)$ is associated with the preference saliency parameter $\left(\theta_{\text {preference }}\right)$. (B) Data driven approach: The behavioral preference saliency measure was associated with the perceptual one.

\section{Discussion}

In this study we have modified the classic Navon task so that subjects freely focus on both of the processing levels while they make a magnitude comparison between the levels (in the numerical cognition task) or while they combine the information across levels (representing gain/loss of a mixed gamble lottery) to form a value estimate (in the preference task). We designed this novel task to measure participant's own (spontaneous) processing style that is not masked by attentional control differences (responding to the task-relevant dimension and ignoring the other one).

We showed that in both tasks there is a high variability in global/local bias across subjects, which is in line with previous studies (Dale \& Arnell, 2010, 2013, 2015; De-Wit \& Wagemans, 2013). Most subjects showed a global bias, that is, preference for information nested at the global level, whereas some subjects had no bias at all, and some demonstrated a local bias, that is, an enhanced ability for processing lowlevel information. This high variability across subjects probably contributed to our small group average effects. We found a small average global bias, in which subjects tend to accept the lottery when the global level is a gain compared to when the global level is a loss. Importantly, the global bias was evident even after controlling for the effect of EV on choice, demonstrating an effect of perceptual processing on value-based choice (Anderson, 2013; Glickman et al., 2019; Hickey, Chelazzi, \& Theeuwes, 2010; Milosavljevic, Navalpakkam, Koch, \& Rangel, 2012; Towal, Mormann, \& Koch, 2013).

These findings emphasize the importance of not only relying on group averages but rather examining heterogeneity across subjects. Approximately a third of the subjects demonstrate a local bias, which is 
in contrast to our finding for the group and is opposite to the common finding reported in the literature (Flevaris \& Robertson, 2016; Kimchi, 1992; Navon, 1977; Wagemans, Feldman, et al., 2012).

In the numerical cognition task, we found that subjects were also affected by the numerical distance of the numbers, as has been shown in previous studies (Dehaene, Dupoux, \& Mehler, 1990; Moyer \& Landauer, 1967). They were faster and more accurate as the distance between the numbers increased. Moreover, on average, subjects showed a small tendency for global precedence (which in our task was the same as a congruency effect) in which they tend to be more accurate and faster when the global digit was larger than the local digit, compared to the opposite. This is in line with previous studies that demonstrated a numerical congruency effect (Dehaene, 2011; Dehaene et al., 1990; Henik \& Tzelgov, 1982; Moyer \& Landauer, 1967), but we substituted the dimension of size with global/local presentation. Across subjects there was no correlation between loss-aversion and saliency parameters, suggesting that these effects probably result from different underlying mechanisms. Although, previous studies demonstrated the positive effect of saliency in increasing the propensity to choose a more salient option with equal value (Milosavljevic et al., 2012; Towal et al., 2013), and that previously rewarded locations are attended to faster and more accurate (Anderson, 2013; Hickey et al., 2010), our findings suggest that on a subject level, loss aversion is not directly influenced by our saliency manipulation and that global/local processing tendency is not directly linked to the mechanisms inducing loss aversion.

Our central result showed that there is a high correlation between the local/global measures in the two tasks. The high correlation across tasks indicates that global/local processing style within an individual is robust when the goal of the task changes. We suggest that the robust correlation that we found was due to our novel design in which subjects were free to focus on either of the processing levels, revealing their "true" subjective trait regarding their processing style, irrespective of the specific task.

These robust correlations hold whether we use simple behavioral measures or whether we use the parameters extracted from our computational modelling (Fig. 5A-B). Using the latter framework allowed us to decompose task-performance measures (accuracy and response-times) into interpretable psychological processes, to isolate the effects of saliency in the numerical cognition and value-based tasks, and to examine the association between them. In particular, we modeled the value-based task using a logit model, in which the amounts were modulated by global precedence. The numerical cognition task was modeled using a biologically plausible neural network based on a place-coding system (Verguts et al., 2005), and in which the activations in the input field are modulated by global precedence.

To conclude, we demonstrated a robust measure of global/local individual differences, which we suggest to be crucial in order to examine complex cognitive mechanisms and their influence across domains. Such processing differences in global/local processing are likely to account for some of the variability in a variety of cognitive tasks, ranging from perception, memory, decision-making, and creativity, as well to mediate a number of cognitive/clinical pathologies like autism and schizophrenia.

\section{References}

Anderson, B. A. (2013). A value-driven mechanism of attentional selection. Journal of Vision. https://doi.org/10.1167/13.3.7

Bellgrove, M. A., Vance, A., \& Bradshaw, J. L. (2003). Local-global processing in earlyonset schizophrenia: Evidence for an impairment in shifting the spatial scale of attention. 
Brain and Cognition. https://doi.org/10.1016/S0278-2626(02)00509-2

Caparos, S., Ahmed, L., Bremner, A. J., de Fockert, J. W., Linnell, K. J., \& Davidoff, J. (2012). Exposure to an urban environment alters the local bias of a remote culture. Cognition. https://doi.org/10.1016/j.cognition.2011.08.013

Caparos, S., Linnell, K. J., Bremner, A. J., de Fockert, J. W., \& Davidoff, J. (2013). Do Local and Global Perceptual Biases Tell Us Anything About Local and Global Selective Attention? Psychological Science. https://doi.org/10.1177/0956797612452569

Chamberlain, R., McManus, I. C., Riley, H., Rankin, Q., \& Brunswick, N. (2013). Local processing enhancements associated with superior observational drawing are due to enhanced perceptual functioning, not weak central coherence. Quarterly Journal of Experimental Psychology. https://doi.org/10.1080/17470218.2012.750678

Chamberlain, R., Van der Hallen, R., Huygelier, H., Van de Cruys, S., \& Wagemans, J. (2017). Local-global processing bias is not a unitary individual difference in visual processing. Vision Research. https://doi.org/10.1016/j.visres.2017.01.008

Dale, G., \& Arnell, K. M. (2010). Individual differences in dispositional focus of attention predict attentional blink magnitude. Attention, Perception, \& Psychophysics. https://doi.org/10.3758/APP.72.3.602

Dale, G., \& Arnell, K. M. (2013). Investigating the stability of and relationships among global/local processing measures. Attention, Perception, \& Psychophysics. https://doi.org/10.3758/s13414-012-0416-7

Dale, G., \& Arnell, K. M. (2015). Multiple measures of dispositional global/local bias predict attentional blink magnitude. Psychological Research. https://doi.org/10.1007/s00426014-0591-3

Davidoff, J., Fonteneau, E., \& Fagot, J. (2008). Local and global processing: Observations from a remote culture. Cognition. https://doi.org/10.1016/j.cognition.2008.06.004

De-Wit, L., \& Wagemans, J. (2013). Individual differences in local and global perceptual organization. In J. Wagemans (Ed.), Oxford Handbook of Perceptual Organization (pp. 713-735). Oxford, UK: Oxford University Press.

De Martino, B., Harrison, N. A., Knafo, S., Bird, G., \& Dolan, R. J. (2008). Explaining enhanced logical consistency during decision making in autism. Journal of Neuroscience. https://doi.org/10.1523/JNEUROSCI.2895-08.2008

Dehaene, S. (2011). The Number Sense: How the Mind Creates Mathematics, Revised and Updated Edition. In The number sense How the mind creates mathematics rev and updated ed.

Dehaene, S., Dupoux, E., \& Mehler, J. (1990). Is Numerical Comparison Digital? Analogical and Symbolic Effects in Two-Digit Number Comparison. Journal of Experimental Psychology: Human Perception and Performance, 16(3), 626-641. https://doi.org/10.1037/0096-1523.16.3.626

Della Libera, C., \& Chelazzi, L. (2006). Visual selective attention and the effects of monetary rewards. Psychological Science, 17(3), 222-227. https://doi.org/10.1111/j.14679280.2006.01689.x

Dijkstra, K. A., van der Pligt, J., \& van Kleef, G. A. (2013). Deliberation Versus Intuition: Decomposing the Role of Expertise in Judgment and Decision Making. Journal of Behavioral Decision Making. https://doi.org/10.1002/bdm.1759

Drake, J. E., \& Winner, E. (2011). Realistic drawing talent in typical adults is associated with the same kind of local processing bias found in individuals with ASD. Journal of Autism and Developmental Disorders. https://doi.org/10.1007/s10803-010-1143-3

Flevaris, A. V., \& Robertson, L. C. (2016). Spatial frequency selection and integration of global and local information in visual processing: A selective review and tribute to Shlomo Bentin. Neuropsychologia. 
https://doi.org/10.1016/j.neuropsychologia.2015.10.024

Förster, J., \& Higgins, E. T. (2005). How global versus local perception fits regulatory focus. Psychological Science. https://doi.org/10.1111/j.1467-9280.2005.01586.x

Förster, J., Liberman, N., \& Kuschel, S. (2008). The Effect of Global Versus Local Processing Styles on Assimilation Versus Contrast in Social Judgment. Journal of Personality and Social Psychology. https://doi.org/10.1037/0022-3514.94.4.579

Glickman, M., Sharoni, O., Levy, D. J., Niebur, E., Stuphorn, V., \& Usher, M. (2019). The formation of preference in risky choice. PLoS Computational Biology. https://doi.org/10.1371/journal.pcbi.1007201

Hedge, C., Powell, G., \& Sumner, P. (2018). The reliability paradox: Why robust cognitive tasks do not produce reliable individual differences. Behavior Research Methods. https://doi.org/10.3758/s13428-017-0935-1

Henik, A., \& Tzelgov, J. (1982). Is three greater than five: The relation between physical and semantic size in comparison tasks. Memory \& Cognition. https://doi.org/10.3758/BF03202431

Hickey, C., Chelazzi, L., \& Theeuwes, J. (2010). Reward changes salience in human vision via the anterior cingulate. Journal of Neuroscience. https://doi.org/10.1523/JNEUROSCI.1026-10.2010

Kahneman, D., \& Tversky, A. (1979). Prospect Theory: An Analysis of Decision under Risk. Econometrica, 47 (2), 263-292.

Kimchi, R. (1992). Primacy of wholistic processing and global/local paradigm: A critical review. Psychological Bulletin. https://doi.org/10.1037/0033-2909.112.1.24

Lewis, M. B., \& Dawkins, G. (2015). Local Navon letter processing affects skilled behavior: A golf-putting experiment. Psychonomic Bulletin and Review. https://doi.org/10.3758/s13423-014-0702-6

Mevorach, C., Humphreys, G. W., \& Shalev, L. (2006). Opposite biases in salience-based selection for the left and right posterior parietal cortex. Nature Neuroscience. https://doi.org/10.1038/nn1709

Milne, E., \& Szczerbinski, M. (2009). Global and local perceptual style, field-independence, and central coherence: An attempt at concept validation. Advances in Cognitive Psychology. https://doi.org/10.2478/v10053-008-0062-8

Milosavljevic, M., Navalpakkam, V., Koch, C., \& Rangel, A. (2012). Relative visual saliency differences induce sizable bias in consumer choice. Journal of Consumer Psychology. https://doi.org/10.1016/j.jcps.2011.10.002

Moyer, R. S., \& Landauer, T. K. (1967). Time required for judgements of numerical inequality. Nature, 215(5109), 1519-1520. https://doi.org/10.1038/2151519a0

Mumma, G. H. (1993). The embedded figures test: internal structure and development of a short form. Personality and Individual Differences. https://doi.org/10.1016/01918869(93)90029-3

Navon, D. (1977). Forest Before Trees : The Precedence of Global in Visual Perception. Cognitive Psychology, 9, 353-383. https://doi.org/10.1016/0010-0285(77)90012-3

Navon, D. (1981). The forest revisited: More on global precedence. Psychological Research. https://doi.org/10.1007/BF00309635

Palmer, S. E. (1977). Hierarchical structure in perceptual representation. Cognitive Psychology. https://doi.org/10.1016/0010-0285(77)90016-0

Ratcliff, R. (2002). A diffusion model account of response time and accuracy in a brightness discrimination task: Fitting real data and failing to fit fake but plausible data. Psychonomic Bulletin and Review. https://doi.org/10.3758/BF03196283

Ratcliff, R., \& McKoon, G. (2008). The diffusion decision model: Theory and data for twochoice decision tasks. Neural Computation. https://doi.org/10.1162/neco.2008.12-06-420 
Ratcliff, R., \& Rouder, J. N. (1998). Modeling Response Times for Two-Choice Decisions. Psychological Science. https://doi.org/10.1111/1467-9280.00067

Stoesz, B. M., Jakobson, L. S., Kilgour, A. R., \& Lewycky, S. T. (2007). Local processing advantage in musicians: Evidence from disembedding and constructional tasks. Music Perception. https://doi.org/10.1525/mp.2007.25.2.153

Towal, R. B., Mormann, M., \& Koch, C. (2013). Simultaneous modeling of èisual saliency and èalue computation improèes predictions of economic choice. Proceedings of the National Academy of Sciences of the United States of America. https://doi.org/10.1073/pnas.1304429110

Tversky, A., \& Kahneman, D. (1992). Advances in prospect theory: Cumulative representation of uncertainty. Journal of Risk and Uncertainty. https://doi.org/10.1007/BF00122574

Van Eylen, L., Boets, B., Steyaert, J., Wagemans, J., \& Noens, I. (2018). Local and Global Visual Processing in Autism Spectrum Disorders: Influence of Task and Sample Characteristics and Relation to Symptom Severity. Journal of Autism and Developmental Disorders. https://doi.org/10.1007/s10803-015-2526-2

Verguts, T., Fias, W., \& Stevens, M. (2005). A model of exact small-number representation. Psychonomic Bulletin and Review. https://doi.org/10.3758/BF03196349

Wagemans, J., Elder, J. H., Kubovy, M., Palmer, S. E., Peterson, M. A., Singh, M., \& von der Heydt, R. (2012). A century of Gestalt psychology in visual perception: I. Perceptual grouping and figure-ground organization. Psychological Bulletin. https://doi.org/10.1037/a0029333

Wagemans, J., Feldman, J., Gepshtein, S., Kimchi, R., Pomerantz, J. R., Van der Helm, P. A., \& Van Leeuwen, C. (2012). A century of Gestalt psychology in visual perception: II. Conceptual and theoretical foundations. Psychological Bulletin. https://doi.org/10.1037/a0029334

Witkin, H, Oltman, P, Raskin E \& Karp, S. (1971). A Manual for The Group Embedded Figures Test. In Consulting Psychologists Press, Palo Alto, CA.

Zmigrod, S., Zmigrod, L., \& Hommel, B. (2015). Zooming into creativity: Individual differences in attentional global-local biases are linked to creative thinking. Frontiers in Psychology. https://doi.org/10.3389/fpsyg.2015.01647

\section{Acknowledgments}

M.U. and D.J.L. were supported by the United States-Israel Binational Science Foundation (CNCRS = 2014612);

\section{Author Contributions}

MG analyzed the data and wrote the manuscript, TS designed the experiments and collected the data, MU wrote the manuscript, and DJL designed the experiments and wrote the manuscript.

\section{Additional Information (including a Competing Interests Statement)}

All authors declare that they have no competing interests 


\section{Supplementary Information}

Numerical Cognition Task - Best Fitted parameters

Table S1. The best fitted parameters of the numerical cognition task. Values in parentheses correspond to the standard deviation of the best fitted parameters across participants.

\begin{tabular}{|c|c|c|c|}
\hline Subject & Noise & Boundary & Saliency \\
\hline 1 & 0.13 & 0.13 & 0.98 \\
\hline 2 & 0.06 & 0.06 & 1.01 \\
\hline 3 & 0.27 & 0.63 & 1.00 \\
\hline 4 & 0.08 & 0.08 & 1.03 \\
\hline 5 & 0.34 & 0.80 & 1.01 \\
\hline 6 & 0.21 & 0.18 & 0.99 \\
\hline 7 & 0.44 & 1.00 & 0.92 \\
\hline 8 & 0.32 & 0.30 & 1.06 \\
\hline 9 & 0.33 & 0.78 & 0.92 \\
\hline 10 & 0.15 & 0.15 & 1.07 \\
\hline 11 & 0.33 & 0.81 & 0.96 \\
\hline 12 & 0.34 & 0.44 & 1.14 \\
\hline 13 & 0.03 & 0.04 & 1.00 \\
\hline 14 & 0.13 & 0.12 & 1.04 \\
\hline 15 & 0.11 & 0.11 & 1.02 \\
\hline 16 & 0.11 & 0.15 & 0.98 \\
\hline 17 & 0.13 & 0.13 & 0.87 \\
\hline 18 & 0.38 & 0.98 & 1.08 \\
\hline 19 & 0.04 & 0.05 & 1.03 \\
\hline 20 & 0.22 & 0.61 & 1.03 \\
\hline 21 & 0.30 & 0.32 & 0.95 \\
\hline 22 & 0.05 & 0.06 & 1.01 \\
\hline 23 & 0.34 & 0.80 & 1.01 \\
\hline 24 & 0.33 & 0.21 & 0.81 \\
\hline 25 & 0.03 & 0.05 & 1.04 \\
\hline 26 & 0.06 & 0.06 & 1.03 \\
\hline 27 & 0.11 & 0.11 & 1.03 \\
\hline 28 & 0.31 & 0.75 & 1.04 \\
\hline 29 & 0.04 & 0.05 & 1.00 \\
\hline 30 & 0.31 & 0.31 & 1.21 \\
\hline Group-Level & $\begin{array}{c}0.20 \\
(0.13)\end{array}$ & $\begin{array}{c}0.34 \\
(0.32)\end{array}$ & $\begin{array}{c}1.01 \\
(0.07)\end{array}$ \\
\hline
\end{tabular}




\section{Numerical Cognition Task - Response time distributions}

\section{data $\longrightarrow$ model}
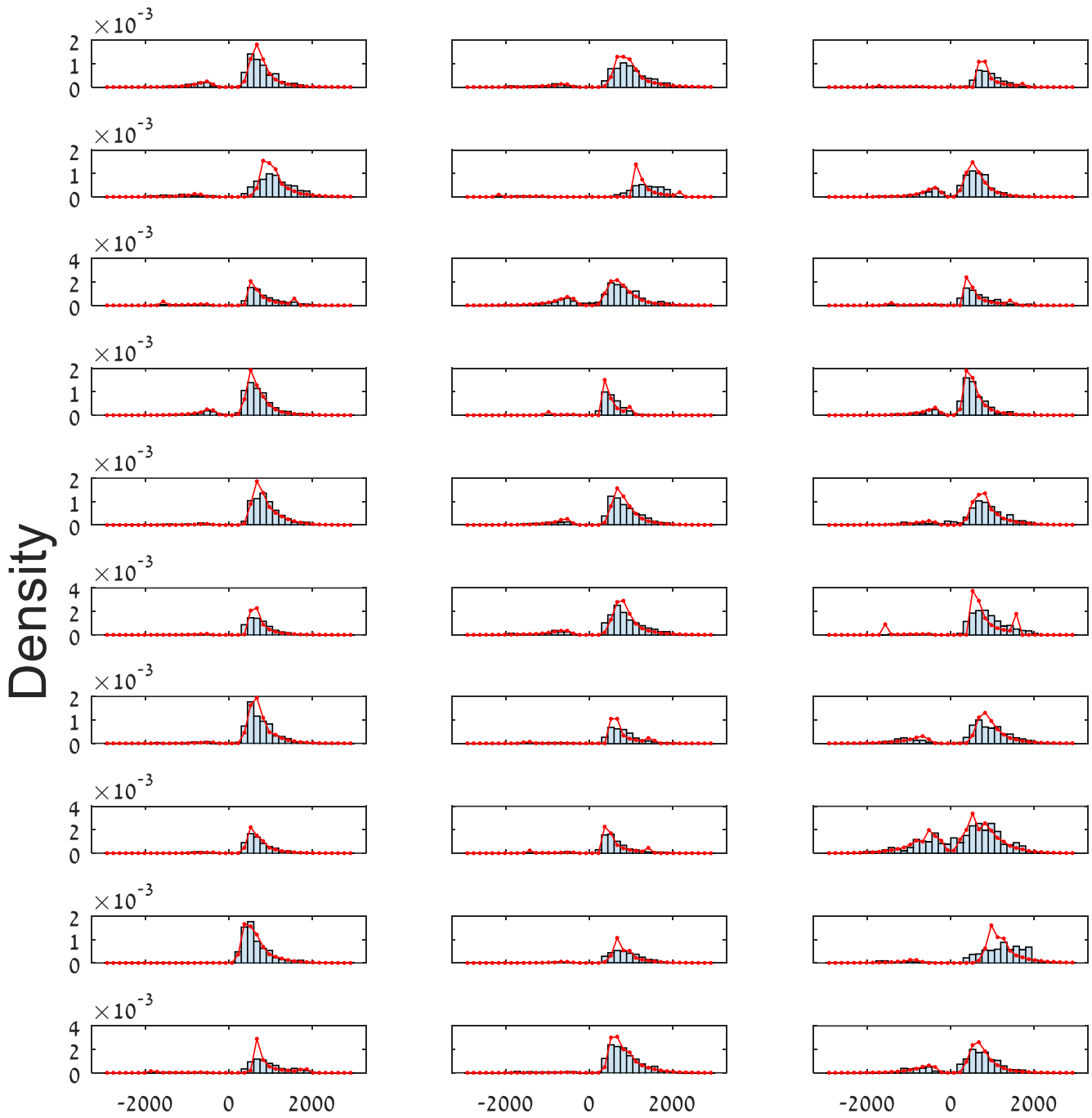

RT (ms)

Fig. S1. Response-Time distributions of all participants in the numerical cognition task. The $x$-axis indicates Reaction-Times, and the y-axis indicates the density. Error responses are mirrored on the negative $x$-axis. Red lines represent simulated RT-distributions for each participant, based on 1,000 simulations of each trial. 
Preference Task - Best Fitted parameters

Table S2. The best fitted parameters of the preference task. Values in parentheses correspond to the standard deviation of the best fitted parameters across participants.

\begin{tabular}{|c|c|c|c|}
\hline Subject & Noise & Loss-Aversion & Saliency \\
\hline 1 & 1.15 & 0.82 & 0.95 \\
\hline 2 & 0.49 & 0.87 & 0.94 \\
\hline 3 & 3.87 & 0.93 & 1.00 \\
\hline 4 & 1.19 & 0.97 & 1.00 \\
\hline 5 & 1.85 & 0.93 & 1.00 \\
\hline 6 & 0.77 & 1.20 & 1.01 \\
\hline 7 & 0.76 & 1.33 & 1.01 \\
\hline 8 & 1.57 & 1.03 & 1.03 \\
\hline 9 & 2.92 & 0.96 & 1.00 \\
\hline 10 & 0.61 & 1.33 & 1.04 \\
\hline 11 & 1.29 & 1.71 & 1.06 \\
\hline 12 & 0.45 & 0.87 & 1.13 \\
\hline 13 & 0.48 & 1.00 & 1.03 \\
\hline 14 & 1.03 & 1.02 & 1.04 \\
\hline 15 & 1.09 & 0.99 & 0.94 \\
\hline 16 & 2.85 & 1.59 & 1.00 \\
\hline 17 & 0.98 & 0.88 & 0.84 \\
\hline 18 & 2.37 & 1.81 & 1.00 \\
\hline 19 & 3.16 & 1.05 & 0.99 \\
\hline 20 & 2.09 & 1.57 & 1.00 \\
\hline 21 & 0.62 & 1.00 & 0.99 \\
\hline 22 & 0.54 & 1.23 & 0.98 \\
\hline 23 & 1.05 & 0.95 & 1.03 \\
\hline 24 & 1.41 & 0.88 & 0.97 \\
\hline 25 & 1.27 & 0.94 & 1.14 \\
\hline 26 & 0.80 & 1.30 & 0.97 \\
\hline 27 & 0.54 & 1.12 & 1.06 \\
\hline 28 & 0.71 & 1.16 & 1.00 \\
\hline 29 & 1.89 & 1.06 & 1.02 \\
\hline 30 & 0.41 & 1.60 & 1.05 \\
\hline Group-Level & $\begin{array}{c}1.34 \\
(0.94)\end{array}$ & $\begin{array}{c}1.13 \\
(0.27)\end{array}$ & $\begin{array}{c}1.01 \\
(0.06)\end{array}$ \\
\hline
\end{tabular}

